\title{
Instability of the Microemulsion Channel in Block Copolymer-Homopolymer Blends
}

\author{
Bart Vorselaars $\odot,{ }^{1}$ Russell K. W. Spencer $\odot{ }^{2}$ and Mark W. Matsen $\oplus^{2, *}$ \\ ${ }^{1}$ School of Mathematics and Physics, University of Lincoln, Brayford Pool, Lincoln LN6 7TS, United Kingdom \\ ${ }^{2}$ Department of Chemical Engineering, Department of Physics \& Astronomy, and Waterloo Institute for Nanotechnology, \\ University of Waterloo, Waterloo, Ontario N2L 3G1, Canada
}

(Received 13 April 2020; accepted 19 August 2020; published 10 September 2020)

\begin{abstract}
Field theoretic simulations are used to predict the equilibrium phase diagram of symmetric blends of $A B$ diblock copolymer with $A$ - and $B$-type homopolymers. Experiments generally observe a channel of bicontinuous microemulsion $(\mathrm{B} \mu \mathrm{E})$ separating the ordered lamellar (LAM) phase from coexisting homopolymer-rich $(A+B)$ phases. However, our simulations find that the channel is unstable with respect to macrophase separation, in particular, $A+B+\mathrm{B} \mu \mathrm{E}$ coexistence at high $T$ and $A+B+\mathrm{LAM}$ coexistence at low $T$. The preference for three-phase coexistence is attributed to a weak attractive interaction between diblock monolayers.
\end{abstract}

DOI: 10.1103/PhysRevLett.125.117801

Diblock copolymers, consisting of an A-type chain joined to a $B$-type chain, are often used to compatibilize immiscible $A$ - and $B$-type homopolymers, in the same way surfactant molecules are use to compatibilize water and oil. In the symmetric case, where copolymers of equal-sized blocks are blended with equal amounts of equal-sized homopolymers, mean-field theory predicts the existence of a Lifshitz critical point [1]. This special type of point [2] has also been predicted for thermotropic liquid crystals [3], ferroelectrics [4], lyotropic liquid crystals [5], magnetic systems [6], polyelectrolytes [7], and biological membranes [8-10], and it generally occurs when the ordered phase of a continuous transition switches from uniform to periodic. The first experimental evidence for Lifshitz critical phenomena was in 1995, when Bates et al. [11] examined ternary blends composed of polyethylene (PE) and polyethylenepropylene (PEP). However, it was understood that the lower critical dimension of the Lifshitz point is four [12-15], and so it should be destroyed by thermal fluctuations. Therefore, Bates et al. hypothesized that fluctuations would push the Lifshitz point to $T=0$ creating a narrow channel of disordered phase. Furthermore, they suggested that the disordered phase inside the channel would become a bicontinuous microemulsion $(\mathrm{B} \mu \mathrm{E})$, where the two homopolymers form interweaving nanosized domains separated by a diblock monolayer, analogous to the traditional $\mathrm{B} \mu \mathrm{E}$ of lyotropic liquid crystals [16]. Bates et al. [17] confirmed the expected behavior two years later. Polymeric microemulsion has since been observed for numerous other chemistries [18-26], and is being studied for a variety of applications including stable polymeric alloys [27], nanoporous functional materials [28], solar cells $[29,30]$, fuel cells [31], and rechargeable batteries [31,32].

Block copolymer melts are generally well described by mean field or rather self-consistent field theory (SCFT), but symmetric ternary blends have become the classic example of where SCFT completely fails. Figure 1 compares the experimental phase diagram of Bates et al. to the SCFT prediction [33-35], where the ratio of homopolymer and copolymer sizes is $\alpha \equiv N_{h} / N_{c}=0.2$ and the Flory-Huggins interaction parameter is $\chi \approx 6.82 / T-0.0118$ [11]. Without copolymer $\left(\phi_{c}=0\right)$, the two homopolymers macrophase separate into coexisting $(A+B)$ phases as the temperature, $T$, is reduced, while neat symmetric diblock copolymer melts $\left(\phi_{c}=1\right)$ microphase separate into an ordered lamellar (LAM) phase. In SCFT, these behaviors are separated by $A+B+$ LAM coexistence, which terminates in a Lifshitz critical point at $\phi_{c, \mathrm{LP}}=2 \alpha^{2} /\left(1+2 \alpha^{2}\right)=0.074 \quad$ [1]

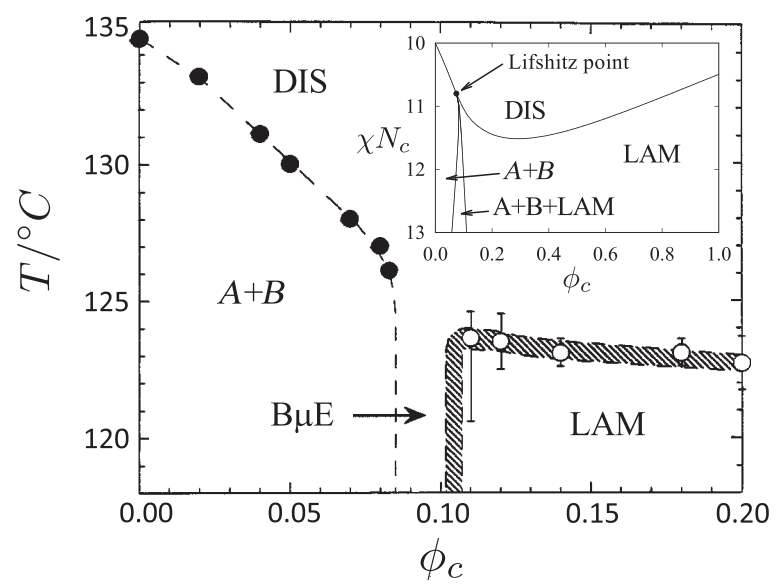

FIG. 1. Experimental phase diagram for $\bar{N}_{c} \approx 2.7 \times 10^{4}$ in term of temperature, $T$, and copolymer volume fraction, $\phi_{c}$, reproduced from Ref. [17]. The experiment observes a microemulsion $(\mathrm{B} \mu \mathrm{E})$ channel, whereas SCFT (see inset) predicts three-phase coexistence between two homopolymer-rich $(A+B)$ phases and an ordered lamellar (LAM) phase. 
whereas in experiments, they are separated by a channel of $\mathrm{B} \mu \mathrm{E}$.

This difference between experiment and SCFT is attributed to fluctuation effects [36,37], which are controlled by the invariant polymerization index of, for example, the copolymer, $\bar{N}_{c}=a^{6} \rho_{0}^{2} N_{c}$, where $a$ is the average segment length and $\rho_{0}$ is the bulk segment density. For the blends of Bates et al., $\bar{N}_{c} \approx 2.7 \times 10^{4}$. In principle, the SCFT phase diagram, corresponding to infinite $\bar{N}_{c}$, should evolve continuously toward the experimental one as $\bar{N}_{c}$ becomes finite, but it is hard to fathom how this could happen. One might imagine that the Lifshitz point and $A+B+$ LAM region shift continuously to lower $T$, ultimately beyond experimental access, leaving behind the $\mathrm{B} \mu \mathrm{E}$ channel. However, this is inconsistent with the fact that the Lifshitz point must vanish once $\bar{N}_{c}$ becomes finite [38]. Using field-theoretic simulations (FTS) [39,40], we generate a theoretical phase diagram for $\bar{N}_{c}=5 \times 10^{4}$ that is free of this inconsistency.

In polymer field theory, a particle-based Gaussian chain model is converted to a field-based model with a Hamiltonian, $H\left[W_{-}, W_{+}\right]$, of the form

$$
\frac{N_{c} H}{\rho_{0} k_{B} T}=\frac{N_{c} H_{Q}}{\rho_{0} k_{B} T}+\int\left(\frac{W_{-}^{2}}{\chi_{b} N_{c}}-W_{+}\right) d \mathbf{r},
$$

where the field $W_{-}(\mathbf{r})$ couples to the difference in $A$ and $B$ concentration $\hat{\phi}_{-}(\mathbf{r})$, the field $W_{+}(\mathbf{r})$ couples to the total concentration $\hat{\phi}_{+}(\mathbf{r})$, and $H_{Q}\left[W_{-}, W_{+}\right]$is the Hamiltonian for an equivalent system of noninteracting polymers acted upon by the fields. SCFT approximates the free energy by $F=H\left[w_{-}, w_{+}\right]$, where $w_{-}(\mathbf{r})$ and $w_{+}(\mathbf{r})$ denote the saddle point of the Hamiltonian, whereas FTS simulate $H\left[W_{-}, W_{+}\right]$with the fields represented on a grid of spacing $\Delta$. One complication is that $W_{+}(\mathbf{r})$ is imaginary valued, which Fredrickson and coworkers handle by performing complex-Langevin FTS [33,41]. We instead use a partial saddle-point approximation for $W_{+}(\mathbf{r})$, whereby $H\left[W_{-}, w_{+}\right]$can be simulated by conventional techniques $[33,34]$. Another problem is an ultraviolet divergence that occurs as $\Delta \rightarrow 0$. Fortunately, the polymer field theory is renormalizable [37], and for large $\bar{N}_{c}$, the divergence can be removed by expressing results in terms of the renormalized interaction parameter [42-44]

$$
\chi=\left(1-\frac{2.333 R_{c}}{\Delta \sqrt{N}_{c}}\right) \chi_{b},
$$

where $R_{c}=a \sqrt{N_{c}}$ is the natural end-to-end length of the copolymer molecules.

The first application of FTS was by Düchs et al. [33,34], but those studies were limited to 2D. More recently, Spencer and Matsen [45] performed Monte Carlo simulations of $H\left[W_{-}, w_{+}\right]$in $3 \mathrm{D}$. By adapting the FTS to a

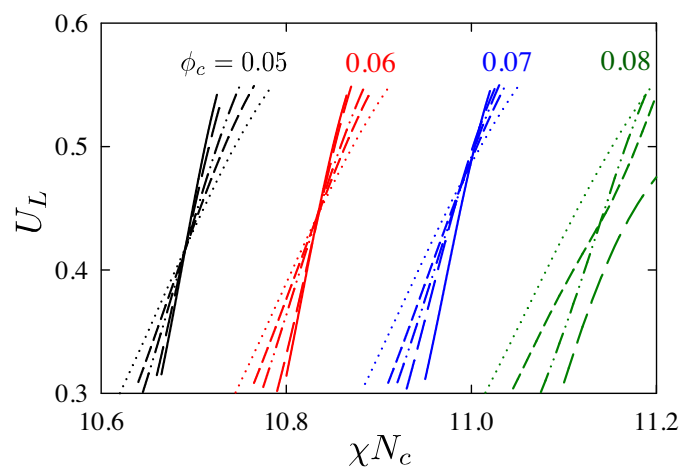

FIG. 2. Cumulants, $U_{L}$, for compositions of $\phi_{c}=0.05,0.06$, 0.07, and 0.08, using semi-grand-canonical FTS for simulation boxes of $m=12$ (dotted), 14 (short dash), 16 (dash-dotted), 20 (long dash), and 24 (solid), where $\Delta=0.168 R_{c}$.

variety of different ensembles, they found evidence for $A+B+\mathrm{B} \mu \mathrm{E}$ coexistence, but the high computational costs limited the size of their simulations boxes and resulted in significant metastability issues. Since then, however, Beardsley et al. [46] found a speedup of about two orders of magnitude by switching to conventional Langevin simulations. With this improvement in hand, we revisit the ternary blends. All simulations are performed in cubic boxes of various sizes, $L=m \Delta$.

We first locate the Scott line separating $A+B$ coexistence from the DIS state, using the finite-size scaling method [47] detailed in Ref. [45]. This method calculates the fourth-order cumulant,

$$
U_{L}=1-\frac{\left\langle\phi_{-}^{4}\right\rangle}{3\left\langle\phi_{-}^{2}\right\rangle^{2}}
$$

of $\phi_{-} \equiv V^{-1} \int \hat{\phi}_{-}(\mathbf{r}) d \mathbf{r}$ with semi-grand-canonical FTS; a sample of the results is plotted in Fig. 2. For blend compositions of $\phi_{c} \lesssim 0.07$, the curves of $U_{L}$ vs $\chi N$ for different $L$ cross at a common fixed point, which identifies the critical point, $\left(\chi N_{c}\right)_{c}$. We also perform the usual check $[43,44]$ that the curves collapse when the $\chi N_{c}$ axis is scaled by $L^{1 / \nu}$, where $\nu=0.63$ is the 3D-Ising exponent. However, for $\phi_{c} \gtrsim 0.08$, the finite-size scaling fails. This anomalous behavior, also encountered in Ref. [45], implies that we have overrun the end of the Scott line. We, therefore, conclude that it ends at an ordinary tricritical point near $\phi_{c} \approx 0.07$. In principle, a finite-size scaling analysis could locate the tricritical point [48], but this would require far larger system sizes and better statistics, which is unfeasible. Nevertheless, further confirmation of the tricritical point will come when we reexamine the $A+B+\mathrm{B} \mu \mathrm{E}$ coexistence.

Next, the transition between LAM and DIS is located using canonical FTS, following the strategy devised in Ref. [49] for neat diblock melts. It involves simulating a large number of replicas at a series of $\chi N$ values spanning 


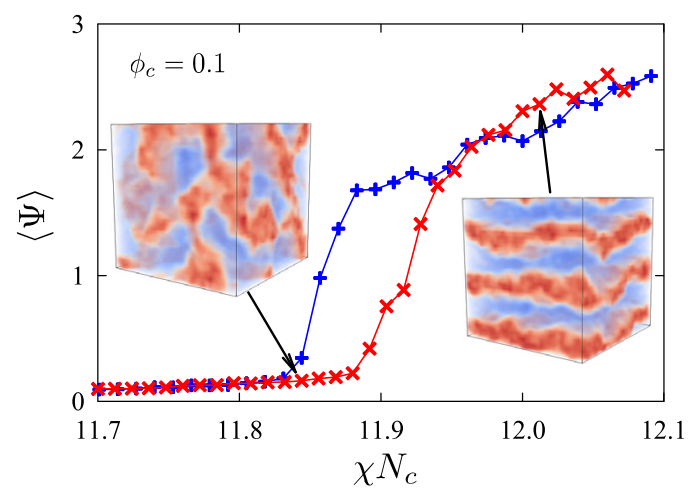

FIG. 3. Order parameter, $\langle\Psi\rangle$, for $\phi_{c}=0.1$, obtained from canonical FTS initialized with DIS $(\times s)$ and LAM $(+s)$ configurations. The images show final configurations with a simulation box of $m=32$ and $L=12.9 R_{c}$.

the transition, where occasional swaps between neighboring replicas are performed using parallel tempering. To track the phase of each replica, we monitor the order parameter, $\langle\Psi\rangle$, where angle brackets denote a short ensemble average over

$$
\Psi=V^{-2} \max _{\mathbf{k}}\left[W_{-}(\mathbf{k}) W_{-}(-\mathbf{k})\right] .
$$

Note that $W_{-}(\mathbf{k})$ is the Fourier transform of $W_{-}(\mathbf{r})$. Figure 3 shows results from a pair of runs at $\phi_{c}=0.1$, one initialized with DIS configurations and another with LAM configurations. At small $\chi N$, both runs converge to low values of $\langle\Psi\rangle$ characteristic of the DIS phase, and at large $\chi N$, to high values characteristic of the LAM phase. This is confirmed by examination of the final configurations. Note that the simulation box was intentionally set to three lamellar periods, based on an estimate of the equilibrium period from a separate simulation of the LAM phase using the box move introduced in Ref. [49]. The fact that the DIS phase spontaneously ordered into three periods confirms our estimate. The DIS-to-LAM transition is bracketed by the narrow metastability interval, $11.83 \lesssim \chi N_{c} \lesssim 11.93$, where both runs remain in their initial phases. For our smallest $\phi_{c}=0.085$, the swelling of the LAM phase limited simulations to two periods, but nevertheless past studies have shown that finite-size effects remain modest even for two periods [49-51], and our current simulations support this.

The transition between $A+B$ and LAM is located following a similar approach [45], but with grand-canonical FTS for replicas at different chemical potentials, $\mu_{c}$, spanning the transition. Parallel tempering was disabled because it becomes ineffective due to the large spacing between replicas. This time, the phase was monitored using short ensemble averages of the copolymer concentration, $\left\langle\phi_{c}\right\rangle$. Figure 4(a) shows results from a pair of independent runs at $\chi N_{c}=11.55$, one initialized with homopolymerrich configurations and another with DIS configurations. At
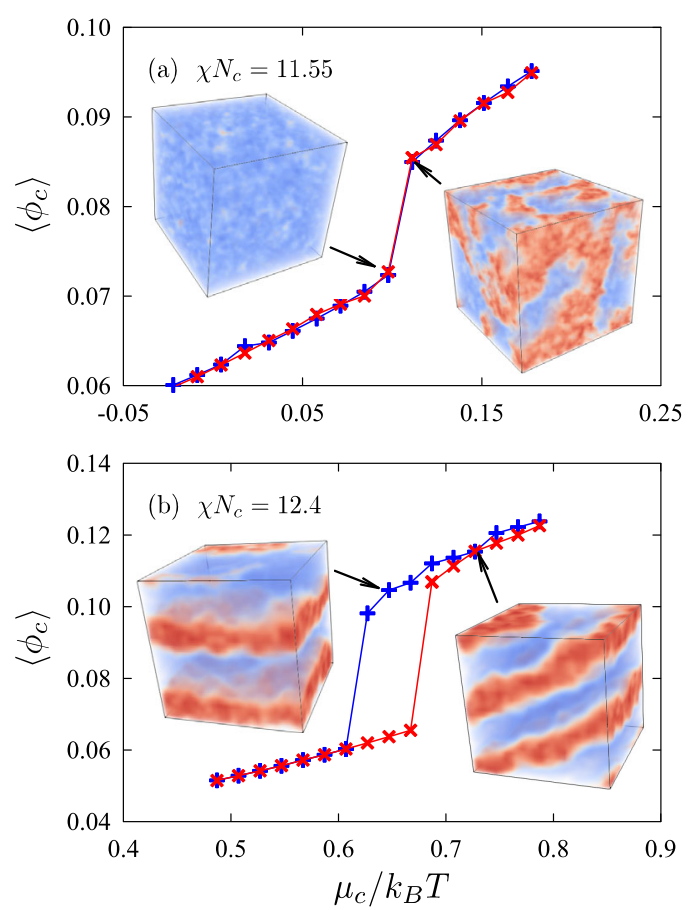

FIG. 4. Average copolymer concentration, $\left\langle\phi_{c}\right\rangle$, for (a) $\chi N_{c}=11.55$ and (b) $\chi N_{c}=12.4$, obtained from grandcanonical FTS initialized with $A$-rich $(\times \mathrm{s})$ and DIS $(+\mathrm{s})$ configurations. The imagines show final configurations with a simulation box of $m=48$ and $L=16.1 R_{c}$.

small $\mu_{c}$, both runs converge to homopolymer-rich phases, and at large $\mu_{c}$, to $\mathrm{B} \mu \mathrm{E}$, as confirmed by inspection of the final configurations. The jump in $\left\langle\phi_{c}\right\rangle$ implies an $A+B+$ $\mathrm{B} \mu \mathrm{E}$ coexistence region.

At the higher segregation of $\chi N_{c}=12.4$ in Fig. 4(b), the replicas initialized with DIS configurations first order into LAM and then switch to homopolymer-rich configurations if $\mu_{c}$ is small. Interestingly, the lamellae tilt at higher $\mu_{c}$ in order to reduce the lamellar period. If $\mu_{c}$ is large, the replicas initialized with a homopolymer-rich phase spontaneously order into LAM. Thus, there is again a jump in $\left\langle\phi_{c}\right\rangle$, but this time it implies $A+B+$ LAM coexistence. Indeed, the intersection of the DIS-to-LAM transition with the three-phase region requires the switch from $A+B+$ $\mathrm{B} \mu \mathrm{E}$ to $A+B+\mathrm{LAM}$.

Our results are summarized by the phase diagram in Fig. 5. The effect of fluctuations is not as extreme as suggested by the experiments. There is a small shift in the Scott line consistent with the prediction for binary homopolymer melts $[43,44,52]$, and a considerably larger shift in the DIS-to-LAM transition similar to that predicted for neat diblocks $[36,42,53]$. The three-phase coexistence of the SCFT phase diagram still remains, but the DIS-to-LAM line intersects it part way down on account of its larger shift. This divides the three-phase coexistence into an $A+B+\mathrm{B} \mu \mathrm{E}$ region at high $T$ and an $A+B+\mathrm{LAM}$ region at low $T$. 


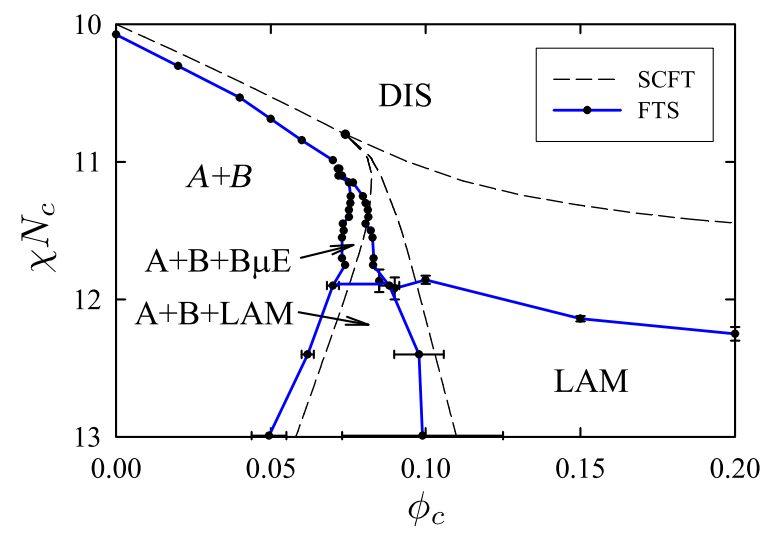

FIG. 5. Comparison of the FTS phase diagram for $\bar{N}_{c}=$ $5 \times 10^{4}$ to the SCFT prediction for $\bar{N}_{c} \rightarrow \infty$.

Experiments proposed the $\mathrm{B} \mu \mathrm{E}$ channel in Fig. 1 when it was mistakenly thought that the $A+B$ and LAM regions of the SCFT phase diagram were separated by an unbinding transition $[11,17]$. In this case, the experimental phase diagram converges to SCFT by shrinking the width of the channel to zero as $\bar{N}_{c} \rightarrow \infty$, but this convergence is no longer possible for the corrected SCFT diagram with its $A+B+$ LAM coexistence. However, the FTS phase diagram in Fig. 5 nicely resolves this problem. The $A+B+\mathrm{B} \mu \mathrm{E}$ region simply shrinks into the meanfield Lifshitz point as $\bar{N}_{c} \rightarrow \infty$, while all the other FTS phase boundaries shift continuously towards their SCFT counterparts.

It is useful to contemplate the reason for the mistaken SCFT prediction, which is related to the effective interaction between the diblock monolayers. The unbinding transition would occur if the interaction remained repulsive at large separations, but in fact the interaction energy has a minimum at a finite separation [35,54]. Once the LAM phase swells to this preferred monolayer spacing, any excess homopolymer should macrophase separate resulting in $A+B+$ LAM coexistence. This behavior was initially missed because the energy minimum is extraordinarily shallow, but nevertheless it most certainly exists. In fact, it is intimately related to autophobic dewetting of homopolymer from chemically identical brushes [55], a phenomena that is well documented by experiments [56,57].

Given the weakness of the monolayer interactions, it is understandable why experiments concluded the existence of a $\mathrm{B} \mu \mathrm{E}$ channel. They are performed by equilibrating samples of the DIS phase at a given $\phi_{c}$, and then looking for a transition as $T$ is slowly decreased. For compositions near $\phi_{c} \approx 0.9$ in Fig. 1, DIS samples showed no evidence of a transition down to $119^{\circ} \mathrm{C}$, and micrographs revealed uniform $\mathrm{B} \mu \mathrm{E}$ after 30 mins of annealing. According to Fig. 5, the system should encounter a three-phase region, where the $\mathrm{B} \mu \mathrm{E}$ expels excess homopolymer into $A$ - and $B$ rich regions. As usual, this would occur by nucleation and
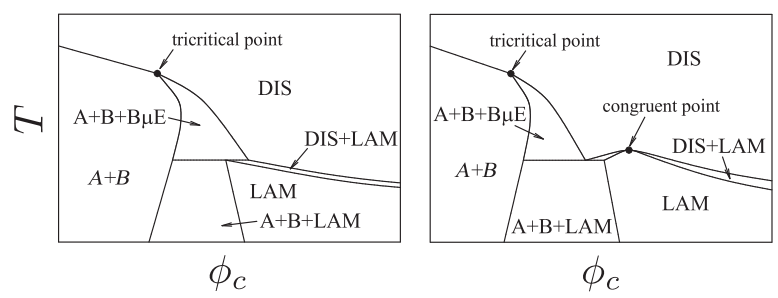

FIG. 6. Topology of the phase diagram for a DIS-to-LAM transition with (a) negative slope and with (b) a reversal in slope.

growth, which is naturally a slow process but even more so in this case. The weak monolayer interactions imply that the free energy gain of macrophase separation is exceptionally small, which in turn implies an unusually large critical nucleus size for the homopolymer-rich domains. Thus, it should be of little surprise that macrophase separation fails to occur in the typical time scale of an experiment.

One might expect that simulations would have discovered the three-phase coexistence years ago, given the intense interest in polymeric $\mathrm{B} \mu \mathrm{E}$. Müller and Schick [58] did, in fact, predict it with simulations of the bondfluctuation model prior to the experiments, but for much different parameter values of $\alpha=1$ and $\bar{N}_{c}=145$. Particle-based simulations for relevant parameter values are simply impractical, because they need to be performed in grand-canonical ensembles, where the insertion of large molecules into the system is extraordinarily difficult [59]. Fortunately, this is not a problem in FTS, allowing us to employ a variety of ensembles. Even with this advantage, our FTS were still highly computational; for example, Figs. 2, 3, and 4 required roughly one CPU-core year, four CPU-core years, and two GPU years of computer resources, respectively.

In multicomponent blends, discontinuous transitions lead to macrophase separation, implying that the DIS and LAM phases must be separated by a DIS + LAM coexistence region. Based on grand-canonical FTS, we conclude that its width is $\Delta \phi_{c} \lesssim 0.001$, which is too narrow to resolve. Nevertheless, its qualitative effect on the phase diagram can be deduced from the slope of the DIS-to-LAM boundary. In Fig. 5, the slope appears to switch from negative to positive as the three-phase region is approached, although we cannot rule out the possibility that the slope remains negative. Figure 6 shows schematic phase diagrams for both possibilities.

Xie et al. [32] have, in fact, observed evidence for the three-phase coexistence, and hopefully our predictions will motivate further studies on the stability of the $\mathrm{B} \mu \mathrm{E}$ channel, with perhaps deeper quenches and longer periods of annealing. It may also help to concentrate on smaller polymers with stronger fluctuation effects and faster dynamics, and to increase $\alpha=N_{h} / N_{c}$, so as to reduce the preferred separation between monolayers and 
strengthen the attraction [54,55]. From an academic standpoint, it is always best to understand the true equilibrium behavior, as this is what the system gravitates towards. Even though the $\mathrm{B} \mu \mathrm{E}$ channel may be metastable on the timescales of typical experiments, applications are likely to require stability for far longer periods of time. Our findings will also have relevance to the numerous other systems involving a mean-field Lifshitz point.

We are grateful to Frank Bates, Glenn Fredrickson, Tim Lodge, Dave Morse, Marcus Müller, and Friederike Schmid for valuable discussions. Compute Canada is acknowledged for providing computer resources.

*Corresponding author.

mwmatsen@uwaterloo.ca

[1] D. Broseta and G. H. Fredrickson, J. Chem. Phys. 93, 2927 (1990).

[2] W. Selke, in Phase Transitions and Critical Phenomena, edited by C. Domb and J. L. Lebowitz (Academic, New York, 1992), Vol. 15.

[3] J. L. Chen and T. C. Lubensky, Phys. Rev. A 14, 1202 (1976).

[4] A. Levstik, C. Filipic, P. Prelovsek, R. Blinc, and L. A. Shuvalov, Phys. Rev. Lett. 54, 1567 (1985).

[5] M. Teubner and R. Strey, J. Chem. Phys. 87, 3195 (1987).

[6] V. Bindilatti, C. C. Becerra, and N. F. Oliveira, Jr., Phys. Rev. B 40, 9412 (1989).

[7] J. F. Joanny and L. Leibler, J. Phys. II (France) 51, 545 (1990).

[8] R. Shlomovitz, L. Maibaum, and M. Schick, Biophys. J. 106, 1979 (2014).

[9] S. Sadeghi, M. Müller, and R. L. C. Vink, Biophys. J. 107, 1591 (2014).

[10] F. Schmid, Biochim. Biophys. Acta 1859, 509 (2017).

[11] F. S. Bates, W. W. Maurer, T. P. Lodge, M. F. Schulz, M. W. Matsen, K. Almdal, and K. Mortensen, Phys. Rev. Lett. 75, 4429 (1995).

[12] A. Erzan and G. Stell, Phys. Rev. B 16, 4146 (1977).

[13] R. M. Hornreich, R. Liebmann, H. G. Schuster, and W. Selke, Z. Phys. B 35, 91 (1979).

[14] A. Kudlay and S. Stepanow, Macromol. Theory Simul. 11, 16 (2002).

[15] D. Zappalà, Phys. Lett. B 773, 213 (2017).

[16] Micelles, Membranes, Microemulsions, and Monolayers, edited by W.M Gelbart, A. Ben-Shaul, and D. Roux (Springer-Verlag, New York, 1994).

[17] F. S. Bates, W. W. Maurer, P. M. Lipic, M. A. Hillmyer, K. Almdal, K. Mortensen, G. H. Fredrickson, and T. P. Lodge, Phys. Rev. Lett. 79, 849 (1997).

[18] M. A. Hillmyer, W. W. Maurer, T. P. Lodge, F. S. Bates, and K. Almdal, J. Phys. Chem. B 103, 4814 (1999).

[19] D. Schwahn, K. Mortensen, H. Frielinghaus, and K. Almdal, Phys. Rev. Lett. 82, 5056 (1999).

[20] D. Schwahn, K. Mortensen, H. Frielinghaus, K. Almdal, and L. Kielhorn, J. Chem. Phys. 112, 5454 (2000).
[21] K. Krishnan, K. Almdal, W. R. Burghardt, T. P. Lodge, and F. S. Bates, Phys. Rev. Lett. 87, 098301 (2001).

[22] V. Pipich, D. Schwahn, and L. Willner, Phys. Rev. Lett. 94, 117801 (2005).

[23] B. M. Habersberger, T. M. Gillard, R. J. Hickey, T. P. Lodge, and F. S. Bates, ACS Macro Lett. 3, 1041 (2014).

[24] C. N. Walker, K. C. Bryson, R. C. Hayward, and G. N. Tew, ACS Nano 8, 12376 (2014).

[25] R. J. Hickey, T. M. Gillard, M. T. Irwin, T. P. Lodge, and F. S. Bates, Soft Matter 12, 53 (2016).

[26] R. J. Hickey, T. M. Gillard, M. T. Irwin, D. C. Morse, T. P. Lodge, and F. S. Bates, Macromolecules 49, 7928 (2016).

[27] J. H. Lee, M. L. Ruegg, N. P. Balsara, Y. Zhu, S. P. Gido, R. Krishnamoorti, and M.-H. Kim, Macromolecules 36, 6537 (2003).

[28] B. H. Jones and T. P. Lodge, ACS Nano 5, 8914 (2011).

[29] D. Kipp, O. Wodo, B. Ganapathysubramanian, and V. Ganesan, ACS Macro Lett. 4, 266 (2015).

[30] D. Kipp, J. Mok, J. Strzalka, S. B. Darling, V. Ganesan, and R. Verduzco, ACS Macro Lett. 4, 867 (2015).

[31] J. Shim, F. S. Bates, and T. P. Lodge, ACS Macro Lett. 8, 1166 (2019).

[32] S. Xie, D. J. Meyer, E. Wang, F. S. Bates, and T. P. Lodge, Macromolecules 52, 9693 (2019).

[33] D. Düchs, V. Ganesan, G. H. Fredrickson, and F. Schmid, Macromolecules 36, 9237 (2003).

[34] D. Düchs and F. Schmid, J. Chem. Phys. 121, 2798 (2004).

[35] M. Yadav, F. S. Bates, and D. C. Morse, Macromolecules 52, 4091 (2019).

[36] G. H. Fredrickson and E. Helfand, J. Chem. Phys. 87, 697 (1987).

[37] P. Grzywacz, J. Qin, and D. C. Morse, Phys. Rev. E 76, 061802 (2007).

[38] L. Kielhorn and M. Muthukumar, J. Chem. Phys. 107, 5588 (1997).

[39] G. H. Fredrickson, V. Ganesan, and F. Drolet, Macromolecules 35, 16 (2002).

[40] M. W. Matsen, J. Chem. Phys. 152, 110901 (2020).

[41] E. M. Lennon, K. Katsov, and G. H. Fredrickson, Phys. Rev. Lett. 101, 138302 (2008).

[42] P. Stasiak and M. W. Matsen, Macromolecules 46, 8037 (2013).

[43] R. K. W. Spencer and M. W. Matsen, Macromolecules 49, 6116 (2016).

[44] R. K. W. Spencer and M. W. Matsen, Macromolecules 51, 4747 (2018).

[45] R. K. W. Spencer and M. W. Matsen, J. Chem. Phys. 148, 204907 (2018).

[46] T. M. Beardsley, R. K. W. Spencer, and M. W. Matsen, Macromolecules 52, 8840 (2019).

[47] K. Binder, Phys. Rev. Lett. 47, 693 (1981).

[48] N. B. Wilding and P. Nielaba, Phys. Rev. E 53, 926 (1996).

[49] B. Vorselaars, P. Stasiak, and M. W. Matsen, Macromolecules 48, 9071 (2015).

[50] K. T. Delaney and G. H. Fredrickson, J. Phys. Chem. B 120, 7615 (2016).

[51] A. Arora, D. C. Morse, F. S. Bates, and K. D. Dorfman, Soft Matter 11, 4862 (2015). 
[52] J. D. Willis, T. M. Beardsley, and M. W. Matsen, J. Chem. Phys. 150, 204906 (2019).

[53] J. Glaser, P. Medapuram, T. M. Beardsley, M. W. Matsen, and D. C. Morse, Phys. Rev. Lett. 113, 068302 (2014).

[54] R. B. Thompson and M. W. Matsen, J. Chem. Phys. 112, 6863 (2000).

[55] M. W. Matsen and J. M. Gardiner J. Chem. Phys. 115, 2794 (2001).
[56] Y. Liu, M. H. Rafailovich, J. Sokolov, S. A. Schwarz, X. Zhong, A. Eisenberg, E. J. Kramer, B. B. Sauer, and S. Satija, Phys. Rev. Lett. 73, 440 (1994).

[57] G. Reiter and R. Khanna, Phys. Rev. Lett. 85, 5599 (2000).

[58] M. Müller and M. Schick, J. Chem. Phys. 105, 8885 (1996).

[59] M. Müller and K. Binder, Comput. Phys. Commun. 84, 173 (1994). 